Shanmugasundaram Ganapathy-Kanniappan, $\mathrm{PhD}$ Rani Kunjithapatham, $\mathrm{PhD}$

Michael S. Torbenson, MD

Pramod P. Rao, MD

Kathryn A. Carson, PhD

Manon Buijs, MD

Mustafa Vali, MD

Jean-François H. Geschwind, MD
${ }^{1}$ From the Russell H. Morgan Department of Radiology and Radiological Sciences (S.G.K., R.K., P.P.R., M.B., M.V., J.F.H.G.) and Department of Pathology (M.S.T.), Johns Hopkins University School of Medicine, 600 N Wolfe St, Blalock 545, Baltimore, MD 21287; and Department of Epidemiology, Johns Hopkins Bloomberg School of Public Health, Baltimore, Md (K.A.C.). Received July 26, 2011; revision requested August 23; revision received September 15; accepted October 12; final version accepted October 20. Supported by the Charles Wallace Pratt Research Fund and Abdulrahman Abdulmalik Research Fund. Address correspondence to J.F.H.G. (e-mail: jfg@jhmi.edu).

\title{
Human Hepatocellular
} Carcinoma in a Mouse Model: Assessment of Tumor Response to Percutaneous Ablation by Using Glyceraldehyde-3-Phosphate Dehydrogenase Antagonists ${ }^{1}$

Materials and Methods:

Results:

Conclusion:
To characterize tumor response to percutaneous injection of glyceraldehyde-3-phosphate dehydrogenase (GAPDH) antagonists in a mouse model of human hepatocellular carcinoma (HCC).

Animal experiments were approved by the Johns Hopkins University Animal Care and Use Committee. Luciferase (luc) gene-expressing Hep3B tumor-bearing athymic nude mice were randomly divided into four groups of six mice each. Tumor-specific GAPDH inhibition was achieved by using percutaneous injection of GAPDH antagonists3-bromopyruvate (3-BrPA) or GAPDH-specific short hairpin RNA (shRNA). Tumor response to treatment was assessed by using bioluminescence imaging and analysis of GAPDH function and apoptotic markers (caspase-3, caspase-9, and positive staining for terminal deoxynucleotidyl transferase-mediated deoxyuridine 5-triphospate nick end labeling). HCC samples from 34 patients were obtained from the Johns Hopkins tumor bank, as approved by the Institutional Review Board, for GAPDH expression analysis. Statistical analysis was performed by using a twosample $t$ test or Spearman rank correlation coefficient.

In vitro, 3-BrPA affected Hep3B cell viability (half maximal inhibitory concentration $=0.15 \mathrm{mmol} / \mathrm{L}$ ), and GAPDH shRNA suppressed (45.5\%) colony formation. In vivo, percutaneous injection of GAPDH antagonists into lucHep3B tumors decreased bioluminescence imaging signal and viability $(3-\mathrm{BrPA}, P<.0001$; GAPDH shRNA, $P=$ $.03)$. The 3 -BrPA treatment primarily inhibited GAPDH activity $(74.5 \%)$ compared with its expression (34.3\%), whereas GAPDH shRNA inhibited both activity (60.6\%) and expression (44.4\%). Targeted inhibition of GAPDH by using 3-BrPA or shRNA induced apoptosis. HCC samples from patients demonstrated a strong correlation between GAPDH upregulation and the proto-oncogene $c$-jun expression $(r=0.543, P=.003)$.

Percutaneous injection of GAPDH antagonists induces apoptosis and blocks Hep3B tumor progression, which demonstrates the therapeutic potential of targeting GAPDH in human HCC. 
$\mathbf{H}$ epatocellular carcinoma (HCC), the most common form of primary liver cancer, is the third leading cause of cancer-related deaths worldwide (1). Because of the lack of specific diagnostic markers and the asymptomatic nature of the disease, patients often present with advanced stages of HCC. Surgery, including transplantation, is currently considered the most effective curative treatment for HCC. However, a majority of patients still have a poor prognosis due to tumor recurrence and chemoresistance (2). Among other therapeutic options for HCC, locoregional therapies have the unique advantage of selectively targeting tumors by using image guidance, thereby minimizing systemic toxicity (3). Current locoregional therapies in clinical practice include intraarterial chemoembolization or radioembolization $(4,5)$ and percutaneous (intratumoral) ablative therapies with chemicals or thermal energy (6) used for various cancers (7-9). Thus, locoregional-targeted delivery through a percutaneous approach of a new and potent chemotherapeutic agent could potentially be very effective in achieving tumor ablation. Such an approach may have the additional advantage of easy translation to clinical practice.

\section{Advances in Knowledge}

- Percutaneous injection of glyceraldehyde-3-phosphate dehydrogenase (GAPDH) antagonists can be successfully used to target cellular GAPDH to induce apoptosis in human hepatocellular carcinoma (HCC).

- Tumor response to molecular targeting of GAPDH can be monitored by using bioluminescence imaging in preclinical models and is relevant for other imaging modalities as well.

- In human HCC, GAPDH expression strongly correlates with the proto-oncogene $c$-jun, which is involved in liver tumorigenesis, suggesting that GAPDH could be a potential therapeutic target.
Emergence of a chemoresistant phenotype poses a major challenge to the success of therapeutic intervention in HCC, which necessitates the search for potent anticancer agents as well as sensitive therapeutic targets. A wealth of data indicates that targeting tumor metabolism could represent an attractive potential anticancer strategy because the majority of solid tumors exhibit increased glucose uptake and aerobic glycolysis (10). This altered metabolic phenotype is accomplished by the upregulation of glycolytic enzymes. In human HCC, aerobic glycolysis and altered expression of glycolytic enzymes have already been documented (11). It is therefore apparent that in HCC, glycolytic enzymes remain potential attractive targets for developing anticancer strategies.

Glyceraldehyde-3-phosphate dehydrogenase (GAPDH), a key glycolytic enzyme, has been known to be upregulated during the progression of HCC $(12,13)$. Several reports based on in vitro data indicate that silencing GAPDH by using antisense oligonucleotides (14) or small interfering RNA (15) induces apoptosis or affects cell proliferation. However, there have been no such reports in vivo, to our knowledge. Plausibly, the ubiquitous nature of GAPDH (16) generates very little enthusiasm to consider it as a molecular target for cancer therapy. Here, through an intratumoral-delivery approach by using percutaneous injection, we investigated the therapeutic potential of targeting

\section{Implications for Patient Gare}

- The results of our study underline the therapeutic potential of percutaneous injection of antiglycolytic agents that target the glycolytic enzyme GAPDH; such targeted therapies may eventually allow us to design and develop effective strategies to intervene with tumor progression.

- Further characterization of a dosing regimen will enable us to maximize the tumoricidal effect and improve the clinical outcome.
GAPDH in vivo. Thus, the purpose of our study was to characterize tumor response to percutaneous injection of GAPDH antagonists in a mouse model of human HCC.

\section{Materials and Methods}

\section{Overview of the Experimental Design}

Human HCC cell line luc-Hep3B (stably expressing the luciferase [luc] gene) was used for the evaluation of the antitumor effect of GAPDH inhibition in vitro and in vivo. Tumor growth in vivo and its response to percutaneous injection of GAPDH inhibitors (3-bromopyruvate [3-BrPA] or GAPDH short hairpin RNA [shRNA]) was monitored by using bioluminescence imaging and was further validated by using biochemical and histochemical analyses. Two authors (S.G.K. and R.K.) designed and performed all in vitro and in vivo experiments and data analysis. One author (P.P.R.) helped with the percutaneous and intratumoral injections. Figure 1

Published online

10.1148/radiol.11111569 Content codes: GI BQ

Radiology 2012; 262:834-845

\section{Abbreviations:}

GAPDH = glyceraldehyde-3-phosphate dehydrogenase $\mathrm{HCC}=$ hepatocellular carcinoma

$\mathrm{mRNA}=$ messenger RNA

shRNA = short hairpin RNA

TUNEL = terminal deoxynucleotidyl transferase-mediated deoxyuridine 5-triphospate nick end labeling

3-BrPA = 3-bromopyruvate

\section{Author contributions:}

Guarantors of integrity of entire study, S.G.K., R.K., P.P.R.; study concepts/study design or data acquisition or data analysis/interpretation, all authors; manuscript drafting or manuscript revision for important intellectual content, all authors; manuscript final version approval, all authors; literature research, S.G.K., R.K.; clinical studies, J.F.H.G.; experimental studies, S.G.K., R.K., M.S.T., P.P.R.; statistical analysis, S.G.K., R.K., K.A.C.; and manuscript editing, S.G.K., R.K., M.S.T., P.P.R., K.A.C., J.F.H.G.

\section{Funding:}

This research was supported by the National Institutes of Health (grant UL1 RR 025005).

Potential conflicts of interest are listed at the end of this article. 


\section{Figure 1}

\begin{tabular}{|c|c|c|c|c|}
\hline \multicolumn{5}{|c|}{ Experimental design } \\
\hline & Day 1 & Day 2 & Day 3 & Day 4----------Day 7 \\
\hline Control & $\begin{array}{l}\text { Single i.t injection } \\
\text { of either saline or } \\
\text { control shRNA ( } 40 \\
\mu g \text { in } 100 \mu \text { l of } \\
\text { serum free MEM } \\
\text { medium) }\end{array}$ & $\begin{array}{l}\text { Single i.t injection } \\
\text { of either saline or } \\
\text { control shRNA (40 } \\
\mu \mathrm{g} \text { in } 100 \mu \mathrm{l} \text { of } \\
\text { serum free MEM } \\
\text { medium) }\end{array}$ & \begin{tabular}{|l|} 
Single i.t injection \\
of either saline or \\
control shRNA (40 \\
$\mu \mathrm{g}$ in $100 \mu \mathrm{l}$ of \\
serum free MEM \\
medium)
\end{tabular} & Resting - no treatment \\
\hline $\begin{array}{l}\text { Intratumoral } \\
\text { targeting } \\
\text { of GAPDH }\end{array}$ & $\begin{array}{l}\text { Single i.t injection } \\
\text { of either } 3 \text {-BrPA } \\
\text { ( } 1.75 \mathrm{mM} \text { in saline) } \\
\text { or GAPDH shRNA } \\
(40 \mu g \text { in } 100 \mu \text { of } \\
\text { serum free MEM } \\
\text { medium) }\end{array}$ & \begin{tabular}{|l|} 
Single i.t injection \\
of either 3 -BrPA \\
(1.75 mM in saline) \\
or GAPDH shRNA \\
( $40 \mu \mathrm{g}$ in $100 \mu \mathrm{l}$ of \\
serum free MEM \\
medium)
\end{tabular} & $\begin{array}{l}\text { Single i.t injection } \\
\text { of either } 3-\mathrm{BrPA} \\
(1.75 \mathrm{mM} \text { in saline }) \\
\text { or GAPDH shRNA } \\
(40 \mu \mathrm{g} \text { in } 100 \mu \mathrm{l} \text { of } \\
\text { serum free MEM } \\
\text { medium) }\end{array}$ & Resting - no treatment \\
\hline
\end{tabular}

Figure 1: Schematic diagram shows in vivo experimental design. i.t= intratumoral, MEM = Eagle minimum essential.

is a schematic diagram of the in vivo experimental design. Finally, HCC samples from patients were analyzed, and they demonstrated a strong positive correlation between GAPDH upregulation and the proto-oncogene c-jun expression.

\section{Cell Culture, Plasmids, and Reagents}

Human primary hepatocytes were procured (Lonza Walkersville, Walkersville, Md) and cultured by using a kit (HCM Bulletkit; Lonza Walkersville) according to supplier instructions. Human HCC cell line Hep3B (ATCC, Manassas, $\mathrm{Va}$ ) was cultured as described previously (17). GAPDH-specific shRNA and control shRNA were obtained (OriGene Technologies, Rockville, Md). Unless otherwise mentioned, all chemicals including 3-BrPA and protease and phosphatase inhibitor cocktails were purchased from Sigma Chemical (St Louis, Mo). Antibodies for GAPDH (Santa Cruz Biotechnology, Santa Cruz, Calif), active caspase- 3 and caspase-9 (Cell Signaling Technology, Danvers, Mass), and $\alpha$-fetoprotein (Thermo Scientific, Logan, Utah) were purchased. The detection reagent (ECL Plus; GE Healthcare, Piscataway, NJ) and the necessary materials (GE Healthcare) for chemiluminescent detection of immunoblots were used. D-luciferin potassium salt used as the substrate for bioluminescence imaging was obtained (Gold Biotechnology, St Louis, Mo). For apoptosis analysis, a terminal deoxynucleotidyl transferase-mediated deoxyuridine 5-triphospate nick end labeling (TUNEL) kit was purchased (Millipore, Bedford, Mass).

\section{Generation of Iuc-Hep3B Cells for Bioluminescence Imaging}

For bioluminescence studies, the luciferase reporter plasmid pcDNA 3.1-cytomegalovirus-firefly luciferase was provided by Martin Pomper and was initially generated in Sam Gambhir's laboratory as described (18). Нер3B cells stably expressing luciferase gene were generated by transfecting them with pcDNA 3.1-cytomegalovirus-firefly luciferase plasmid by using a transfection agent (TurboFectin 8.0; OriGene Technologies), followed by clonal selection with G418 (Invitrogen, Grand Island, NY) containing growth medium. Clones expressing highest luciferase activity were selected and used for further studies. For simplicity, these stable cells will be referred hereafter as lucHep3B. For in vitro bioluminescence imaging, cells growing in log phase were seeded into 24 -well plates the day before the experiment.

\section{Cell Viability, Colony Suppression, and GAPDH Activity Assays}

The effect of 3-BrPA on cell viability was determined by quantifying intracellular adenosine triphosphate level by using a kit (CellTiter-Glo assay kit; Promega,
Madison, Wis) and a multilabel plate reader (Victor3; PerkinElmer, Fremont, Calif). In brief, Hep3B cells were plated in 96-well flat-bottomed opaque plates the day before 3-BrPA treatment, and cellular adenosine triphosphate level was determined per the protocol of the manufacturer. Similarly, the effect of GAPDH shRNA was assessed by using colony suppression assay. Hep3B cells growing in log phase were seeded in six-well plates and transfected with either the control shRNA or GAPDH shRNA plasmid by using a transfection agent (Turbofectin 8.0; OriGene Technologies). After 48 hours of transfection, the media were changed and the colonies were grown for a few days and stained for counting. Colony suppression was evaluated as described by Franken et al (19). The colonies were stained with crystal violet and glutaraldehyde and counted independently by two experienced researchers (S.G.K., R.K.). GAPDH activity was measured on the basis of the principle of rate of oxidation of nicotinamide adenine dinucleotide in its reduced form to that of nicotinamide adenine dinucleotide per minute at $25^{\circ} \mathrm{C}$ under controlled assay conditions (20).

\section{Bioluminescence Imaging Signal Correlation with Cell Viability}

The correlation between bioluminescence imaging signal and luc-Hep3B cell viability was assessed as described 
below. First, increasing numbers of lucHep3B cells $\left(0.1-3 \times 10^{5}\right.$ cells per well $)$ were allowed to grow for 24 hours, and the bioluminescence signal was quantified by using bioluminescence imaging. In the second approach, luc-Hep3B cells growing in log phase were plated $\left(1 \times 10^{5}\right.$ cells per well $)$ and treated with increasing concentrations of 3 -BrPA (0.05, 0.1 , and $0.2 \mathrm{mmol} / \mathrm{L}$ and 1.75 $\mathrm{mmol} / \mathrm{L}$ [a previously determined in vivo therapeutic dose (21)]) for 24 hours followed by bioluminescence imaging. For bioluminescence imaging, cells were washed once with phosphate-buffered saline, and D-luciferin was added to a final concentration of $150 \mu \mathrm{g} / \mathrm{mL}$ according to manufacturer recommendation (Xenogen [now Caliper Life Sciences], Hopkinton, Mass), and images were acquired by using a small animal imaging system (IVIS 200; Xenogen). All cellular studies were performed in triplicate wells with at least two different culture preparations.

\section{In Vivo Studies}

Animal studies were performed as approved by the Johns Hopkins University Animal Care and Use Committee. For the in vivo experiments, 6-8-week-old male athymic nude mice (body weight, 25-30 g) were used (Crl:NU-Foxn $1^{\text {nu }}$ strain; Charles River Laboratory, Germantown, Md). Tumor xenografts were initiated in male athymic nude mice with subcutaneous injection of luc-Hep3B cells $\left(4-5 \times 10^{6}\right.$ cells) growing in log phase. These mice served as donors, and tumors with a positive finding at bioluminescence imaging were extracted, minced into approximately $1 \mathrm{~mm}^{3}$, and implanted subcutaneously into the left or right bottom flank of experimental mice for further studies.

\section{Percutaneous Intratumoral Therapy with 3-BrPA or GAPDH shRNA}

Mice with tumor sizes between 150 and $200 \mathrm{~mm}^{3}$ and with a positive finding for luciferase expression were randomly assigned to two control groups (one each for saline and control shRNA) and two treatment groups (3-BrPA and GAPDH shRNA) with six mice per group. Each tumor received, by using intratumoral injection, either the vehicle (saline) or $1.75 \mathrm{mmol} / \mathrm{L} 3$-BrPA (a previously determined in vivo therapeutic dose [21]) at a volume equal to the size of the tumor (determined with ellipsoid volume formula, $\mathrm{V}=l \cdot w^{2} \cdot 0.52$, where $l$ is length and $w$ is width of the tumor). Injections were performed carefully by using a fanning technique once every 24 hours starting at day 1 and ending on day 3 . After day 3 , no further injections were given for the next 4 days, and on day 7 , bioluminescence imaging was performed to assess tumor response. Just before imaging, mice were injected with D-luciferin intraperitoneally (150 mg per kilogram of body weight), and bioluminescent images were acquired by using a small animal imaging system (IVIS 200; Xenogen) at multiple time intervals (1 minute, 5 minutes, and 15 minutes). The mice were then sacrificed, and tumor tissues were subjected to histopathologic and biochemical analyses. RNA interference for GAPDH was performed by using either control shRNA or GAPDH shRNA, where 40 $\mu \mathrm{g}$ of plasmid was mixed in a volume of serum-free Eagle minimum essential media (proportionate to the tumor volume) and injected slowly into the tumor by using a fanning technique. The delivery of such naked shRNA plasmid was performed every 24 hours for 3 days, and the procedures for tumor response monitoring were performed as described previously.

\section{Immunoblotting, Histologic Examination, Immunohistochemistry, and TUNEL Assay}

Immunoblotting was performed as described previously (17). Antibody dilutions and incubation procedures were followed per the instructions of the supplier. For histologic examination, tissues were routinely fixed in $10 \%$ phosphatebuffered formalin (Polysciences, Warrington, Pa), dehydrated with graded ethanol, embedded in wax (Paraplast Plus; McCormick Scientific, Richmond, III), sliced at $5 \mu \mathrm{m}$, mounted on slides, and oven dried and deparaffinized. The tissue slices were subjected to hematoxylin-eosin staining and viewed under a light microscope. Immunohistochemical detection of GAPDH, $\alpha$-fetoprotein, and active caspase- 3 was performed by using a detection kit (HistostainPlus; Invitrogen), following manufacturer protocol. Apoptosis was assessed by using a TUNEL detection kit (Millipore), following manufacturer protocol. Slices were counterstained with 4',6-diamidine-2-phenylindole for nuclei. Finally, tissue slices were covered with cover slips and mounted with antifade reagent (Prolong Gold; Invitrogen) and allowed to dry in the dark. The slides were viewed with fluorescent microscopy, and the images were captured by using a digital camera (Coolpix; Nikon Instruments, Melville, NY).

\section{Patient HCC Samples and Quantitative Real-time Polymerase Chain Reaction}

Human HCC samples and surrounding healthy liver tissues from 34 patients were obtained from the Johns Hopkins tumor bank according to Institutional Review Board approval. Tumor and adjacent nonneoplastic tissues were determined by an experienced pathologist (M.S.T., 10 years of experience as a liver pathologist). Gene expression analysis was performed by using quantitative real-time polymerase chain reaction with a sequence detection system (ABI 7900HT; Applied Biosystems, Bedford, Mass) and a mix (SYBR Green PCR Master Mix; Applied Biosystems). In brief, total RNA was extracted by using Trizol (Invitrogen) followed by cleanup by using the RNeasy kit (Qiagen, Valencia, Calif). A known quantity of RNA $(10 \mu \mathrm{g})$ was then subjected to reverse transcription by using the High Capacity cDNA Reverse Transcription kit (Applied Biosystems). The complementary DNAs thus synthesized were subjected to quantitative real-time polymerase chain reaction for specific gene expression analysis. The primers used for GAPDH and $c$-jun were as follows: 5'-GAGTCAACGGATTTGGTCGT-3' (forward) and 5'-TTGATTTTGGAGGGATCTCG-3' (reverse) for GAPDH and 5' TCCCCTAACCTCTTTTCTGC $3^{\prime}$ (forward) and 5' AACATCGCACTATCCTTTGG $3^{\prime}$ (reverse) for $c$-jun. The internal control primer set $18 \mathrm{~S}$ was used (Applied Biosystems). 


\section{Statistical Analysis}

Data from the control and treated groups were summarized with means \pm standard error of the mean, the distributions were assessed for normality, and the groups were compared by using two-sample $t$ tests. All $P$ values of bioluminescence imaging measurements were presented by using mean \pm standard error of the mean. Analysis was performed by using software (SAS, version 9.2; SAS Institute, Cary, NC). All reported $P$ values were two-sided, and significant difference was set at $P$ less than .05. Spearman rank correlation coefficient was used to analyze the $c$-jun and GAPDH expression in patient HCC samples. An author (K.A.C.) performed statistical analysis of in vitro and in vivo data.

\section{Results}

\section{Targeting GAPDH Affects Hep3B Cell Viability In Vitro}

Hep3B cells showed overexpression of GAPDH (1.5 fold) compared with the normal human primary hepatocytes, which indicates HCC-related upregulation of GAPDH (Fig 2a). Silencing GAPDH through shRNA in Hep3B cells demonstrated marked colony suppression (45.5\%) compared with the control shRNA (Fig 2b). Similarly, inactivation of GAPDH by using 3 -BrPA demonstrated a dose-dependent depletion of intracellular adenosine triphosphate, which led to loss of viability (Fig 2c). Furthermore, immunoblotting revealed a 3 -BrPA-dependent increase in active caspase-3, confirming the induction of apoptosis (Fig 2d). The 3-BrPA treatment inhibited cellular GAPDH activity $(89.6 \%)$ at $0.1 \mathrm{mmol} / \mathrm{L}$ concentration (Fig 2e).

\section{Percutaneous Injection of 3-BrPA or shRNA Affects /uc-Hep3B Tumor Viability}

Results of bioluminescence imaging of luc-Hep3B cells demonstrated a direct correlation between cell viability and signal intensity, as evidenced by cell number-dependent increase and cytotoxicity-related decrease in total

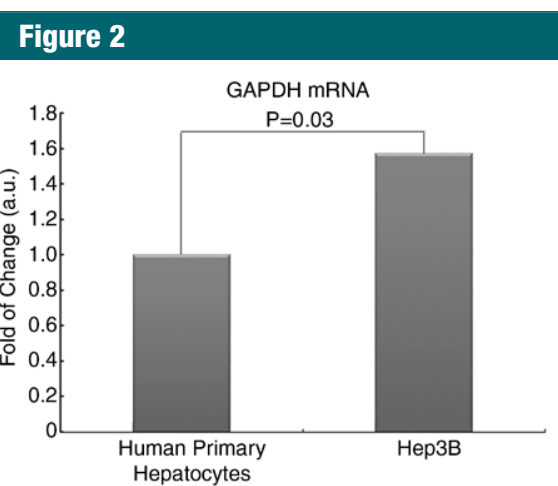

a.
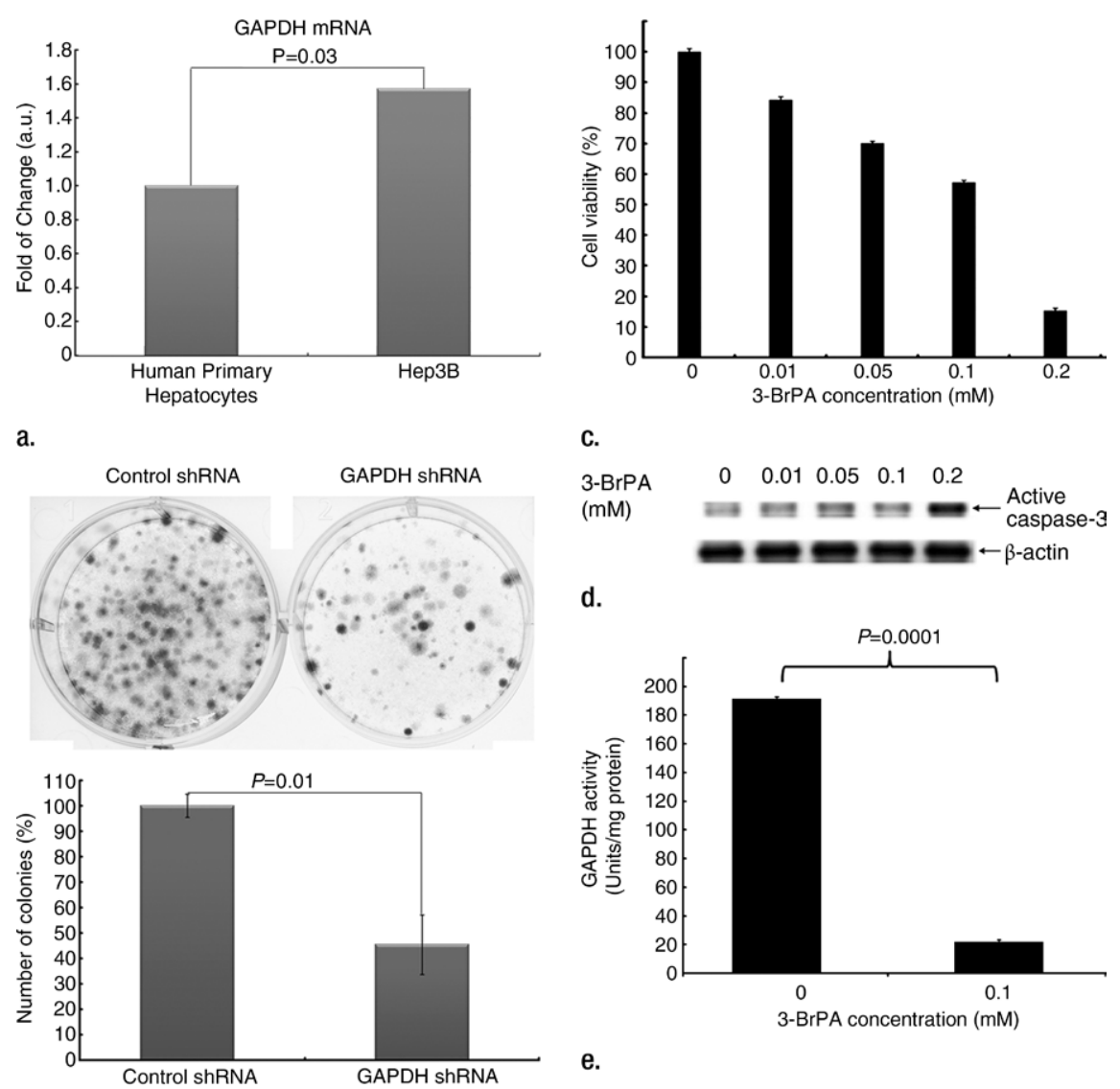

c.

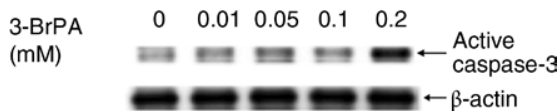

d.

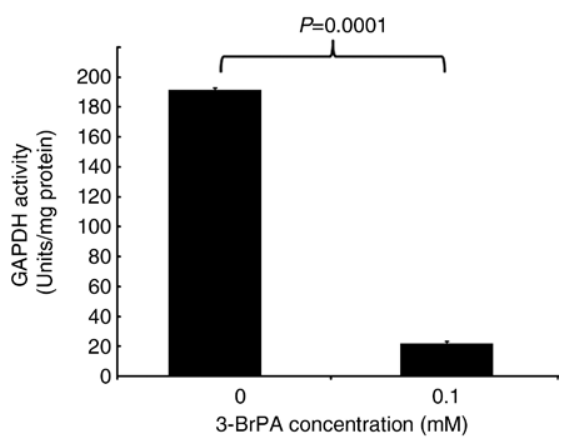

e.

b.

Figure 2: GAPDH antagonists affect cell viability in vitro. (a) Graph shows Hep3B cells with increased level of GAPDH (>1.5 fold) compared with normal human hepatocytes. (b) Graph and photographic images (crystal violet stain) show that silencing GAPDH with shRNA in Hep3B cells significantly suppressed colony formation $(P=.0001)$. (c) Graph shows 3-BrPA treatment for 24 hours resulted in a dose-dependent decrease in intracellular adenosine triphosphate level, indicating loss of viability in Hep3B cells. (d) Immunoblot shows 3-BrPA-dependent induction of active caspase-3 in Hep3B cells. (e) Graph shows 3-BrPA treatment inhibited GAPDH activity (89.6\%) at $0.1 \mathrm{mmol} / \mathrm{L}(\mathrm{mM})$ in Hep3B cells. Error bars = standard error of the mean. a.u. $=$ arbitrary units, $m R N A=$ messenger RNA.

bioluminescence signal (Fig 3). Having validated the correlation between bioluminescence imaging signal and cell viability in vitro, we then monitored the response of the tumor to percutaneous injection of GAPDH antagonists in mice by using bioluminescence imaging. Bioluminescence imaging signals were observed every 24 hours for 4 consecutive days in mice treated with the GAPDH inhibitor 3-BrPA. At the end of the week (day 4 after the final intratumoral delivery), the decrease in bioluminescence imaging signals was stable and statistically significant (Fig 4a, 4b). Immunoblot analysis showed a direct correlation between GAPDH protein level and tumor viability (bioluminescence imaging signal intensity) (Fig 4a, 4c). This was further supported by an increase in the level of active caspase-3 and caspase-9. GAPDH inactivation by the use of percutaneous delivery of 3-BrPA increased the level of phosphorylated heat shock protein 27 , a stress indicator, with a corresponding decrease in phosphorylated Akt (Fig 4c).

Similar to the effect of 3-BrPA, percutaneous injection of GAPDH shRNA 


\section{Figure 3}
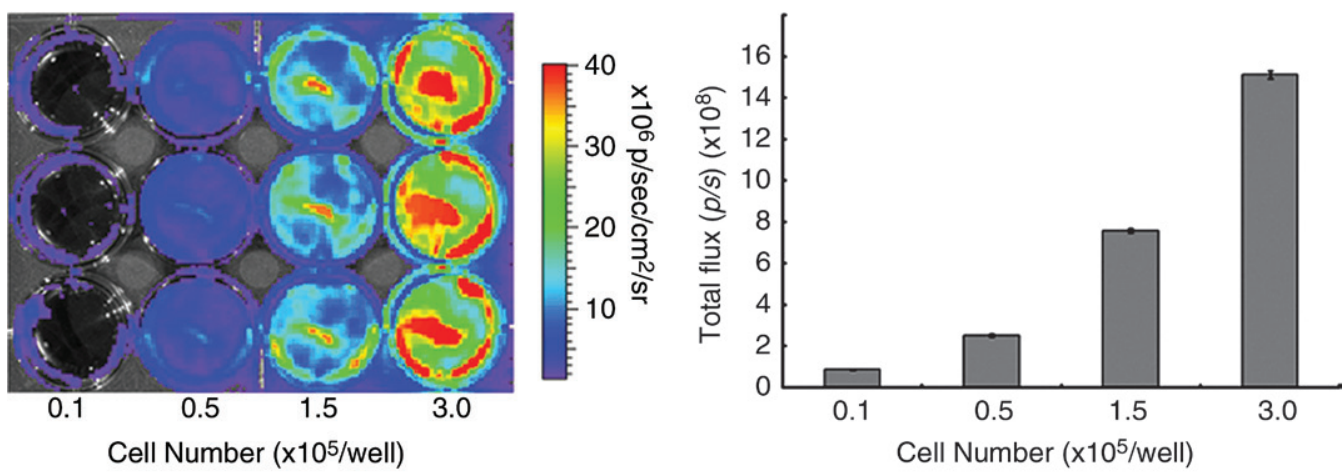

a.

b.
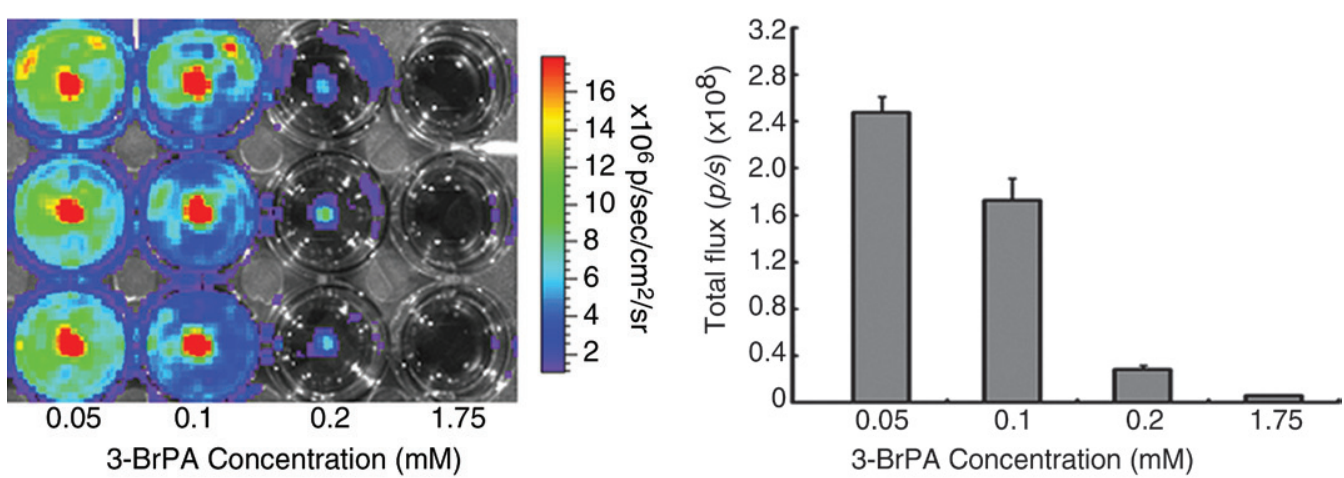

d.

Figure 3: Bioluminescence signal intensity corresponds to cell viability in luc-Hep3B cells. (a) Representative plate of cells imaged. Hep3B cells stably transfected with luciferase (Iuc) gene were plated in increasing numbers and were allowed to grow for 24 hours, followed by bioluminescence imaging. (b) Graph shows mean quantification of bioluminescence imaging $(n=3)$, with cell number-dependent increase in signal intensity of luc-Hep3B cells. (c) Representative plate of cells imaged. luc-Hep3B cells were treated with different concentrations of 3-BrPA, followed by bioluminescence imaging. (d) Graph shows mean quantification of bioluminescence imaging $(n=3)$, with cytotoxicity-dependent decrease in signal intensity. Error bars = standard error of the mean. $p=$ photons, $p / s=$ photons per second, $s r=$ steradian.

demonstrated treatment-dependent decrease in bioluminescence imaging signals (Fig 5a, 5b). Immunoblot analysis of GAPDH shRNA-treated tumors showed a direct correlation between bioluminescence imaging signal and GAPDH protein level (Fig 5a, 5c). This was further supported by an increase in the level of active caspase- 3 and caspase-9. GAPDH knockdown with percutaneous delivery of shRNA caused a decrease in the phosphorylated Akt level, which indicated an effect on the energy sensor pathway. Surprisingly, phosphorylated heat shock protein 27 was not increased (Fig 5c), which indicated a differential stress response between 3-BrPA and shRNA-mediated inhibition of GAPDH.

\section{Percutaneous Injection of 3-BrPA or shRNA Affects GAPDH Activity and Expression}

Data obtained from enzyme assay and quantitative real-time polymerase chain reaction demonstrated a treatment-dependent inactivation of GAPDH and its mRNA depletion in luc-Hep3B tumors. The 3-BrPA-treated tumors revealed a significant decrease in GAPDH activity, with an inhibition level of $74.5 \%$ compared with control tumors (Fig 6a). This decrease in GAPDH activity corroborated the decreasing trend observed in bioluminescence imaging signals (Fig 4b). The 3-BrPA treatment also significantly affected GAPDH mRNA level, with a $34.3 \%$ decrease (Fig 6b). Likewise, GAPDH shRNA-treated tumors showed a significant decrease in GAPDH activity (60.6\%) compared with control tumors (Fig 6c). This decrease in GAPDH activity supports the decreasing trend observed in bioluminescence imaging signals of the GAPDH shRNA-treated tumors (Fig 4b). Furthermore, GAPDH shRNA-injected tumors showed a $44.4 \%$ decrease in total GAPDH mRNA level (Fig 6d).

\section{Percutaneous Injection of GAPDH Antagonists Triggers Apoptosis}

Intratumoral delivery of GAPDH antagonists such as 3-BrPA or GAPDH shRNA demonstrated the induction of cell death (Fig 7a, 7b). Hematoxylin-eosin staining of luc-Hep3B tumor slices showed 


\section{Figure 4}
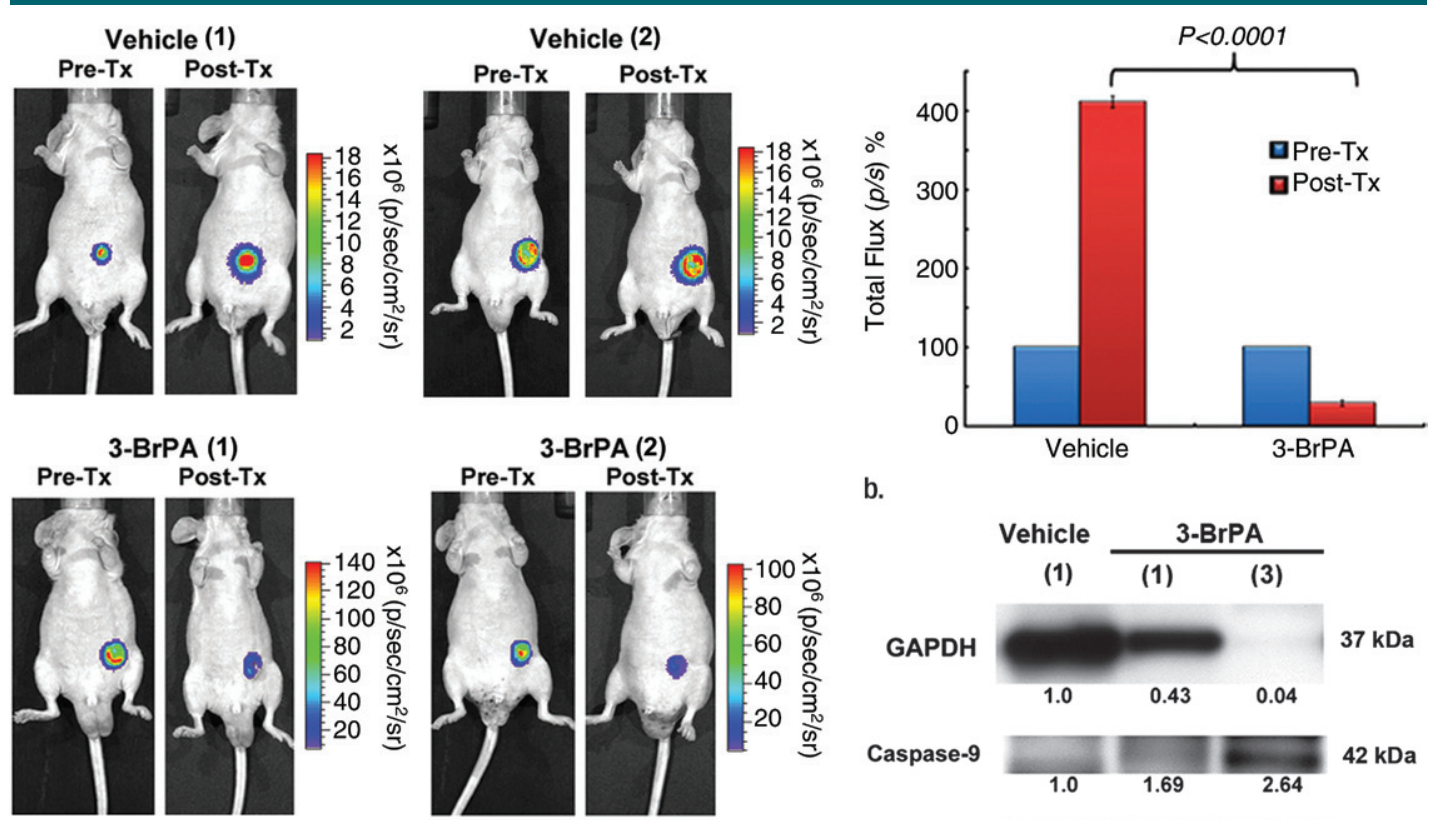

b.

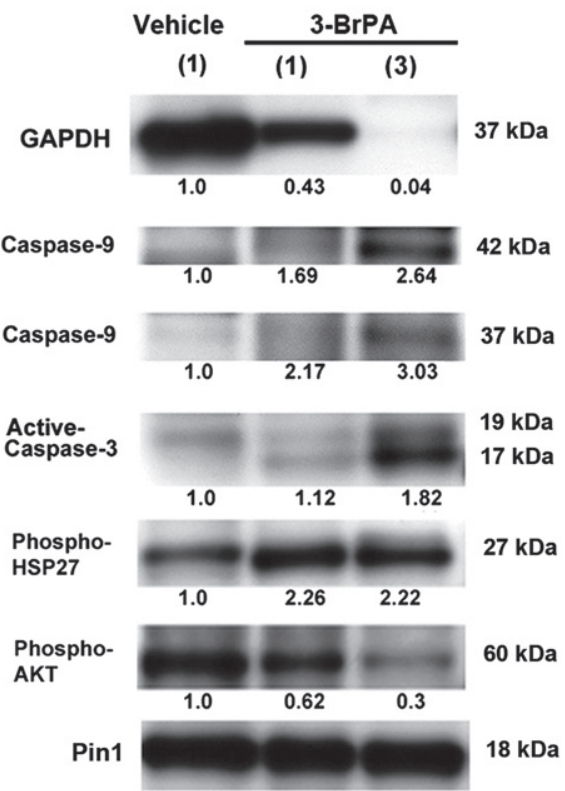

c.

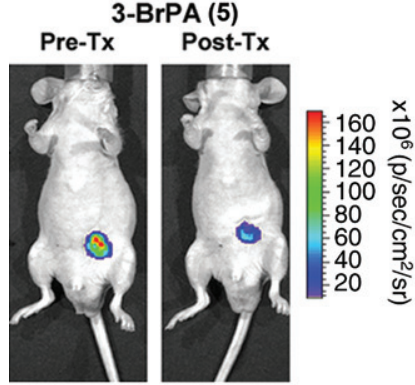

a.

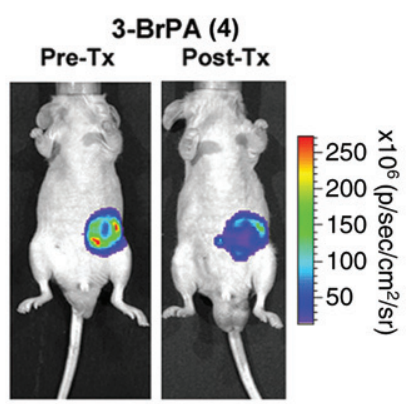

Figure 4: Percutaneous injection of 3-BrPA affects luc-Hep3B tumor in mice. (a) Luc-Hep3B tumor implantation and 3-BrPA (1.75 mmol/L) treatment were followed as described in Materials and Methods section. Bioluminescence images are from representative mice before (Pre- $T x$ ) and after (Post-Tx) treatment. (b) Graph shows mean quantification of bioluminescence imaging signal, with a significant decrease in intensity in 3-BrPA-treated mice $(n=6)$. $P$ value is from two-sample $t$ test comparing groups after treatment. Error bars = standard error of the mean. (c) Immunoblots show luc-Hep3B tumors with 3-BrPA (1.75 mmol/L)-dependent decrease in GAPDH protein level and a corresponding increase in caspase- 9 and active caspase-3 levels. 3-BrPA induced stress response as evidenced by an increase in phosphorylated heat shock protein (HSP) 27 and a corresponding decrease in phosphorylated Akt, an energy sensor. Pin1 = loading control. Numbers in parentheses in the immunoblots (in c) correspond to the respective after treatment (Post-Tx) mouse on bioluminescence images (in a). Numbers at the bottom of blots are densitometry quantifications of respective signals. $p=$ photons, $p / s=$ photons per second, $s r=$ steradian.

viable tumor in the control mice (vehicle and control shRNA), as opposed to the treated mice (3-BrPA or GAPDH shRNA). This treatment-dependent change was further supported by the
TUNEL assay, where only the treated luc-Hep3B tumors showed an abundant green fluorescence indicative of the fragmented DNA, an apoptotic event. Immunohistochemical staining also illustrated a strong positive staining for the apoptotic executioner protein active caspase-3 (brown) only in treated tumors. Notably, the known marker of HCC, $\alpha$-fetoprotein, and 


\section{Figure 5}
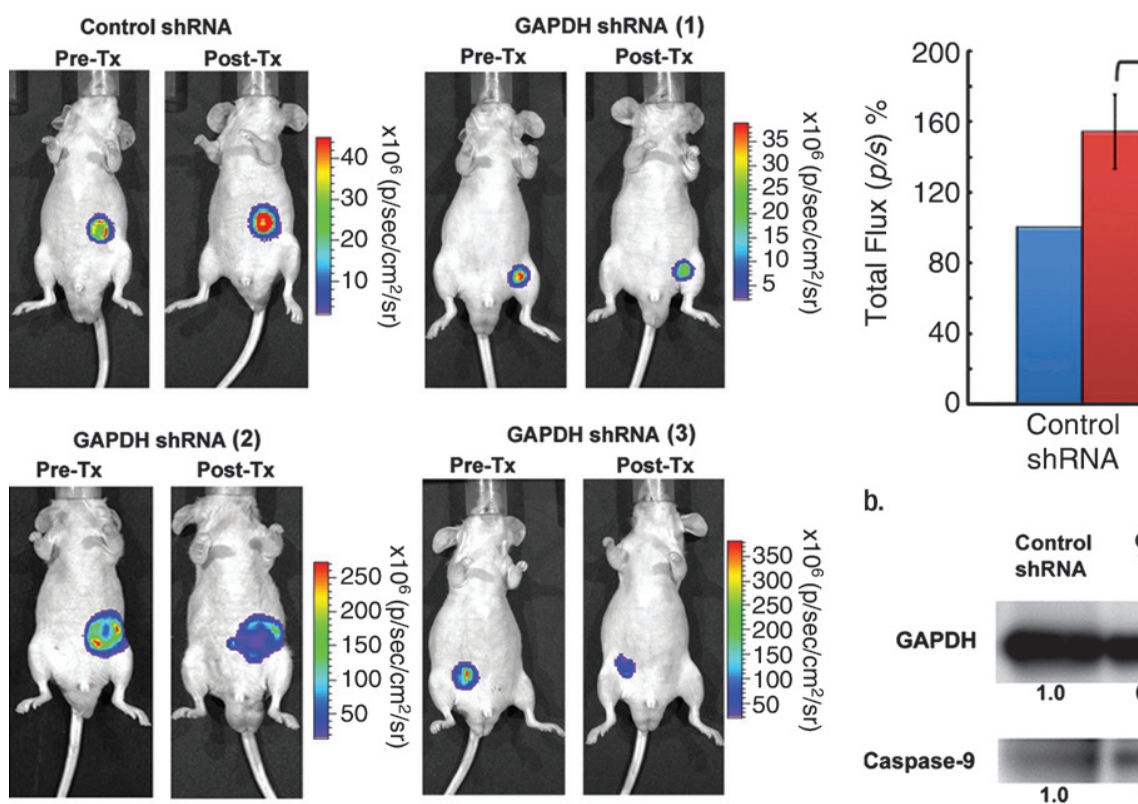

b.

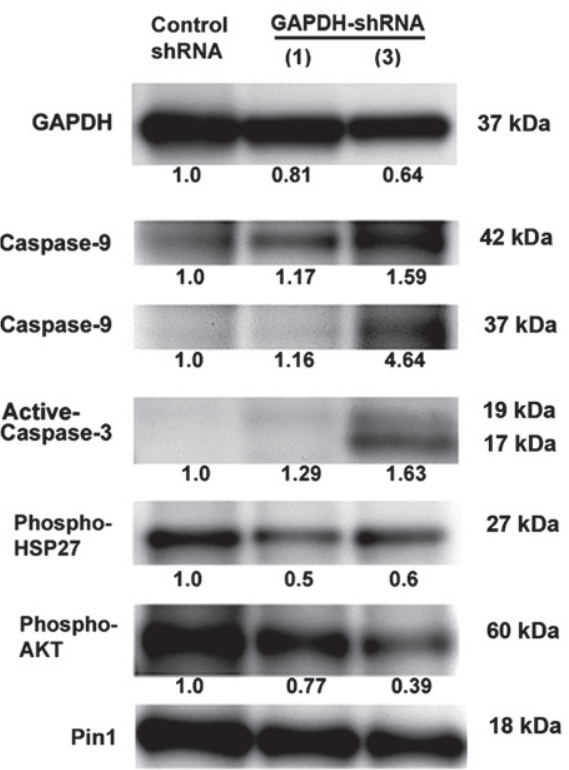

GAPDH shRNA (6)
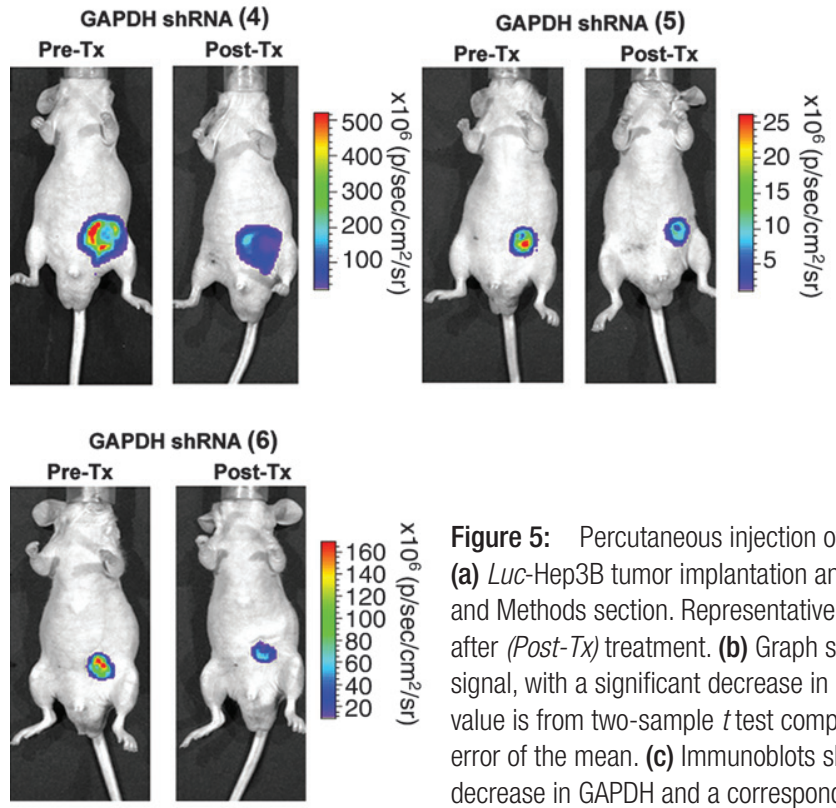

a.

Figure 5: Percutaneous injection of GAPDH shRNA affects luc-Hep3B tumor in mice. (a) Luc-Hep3B tumor implantation and treatment were followed as described in Materials and Methods section. Representative bioluminescence images of mice before (Pre-TX) and after (Post-TX) treatment. (b) Graph shows mean quantification of bioluminescence imaging signal, with a significant decrease in intensity in GAPDH shRNA-treated mice $(n=6)$. $P$ value is from two-sample $t$ test comparing groups after treatment. Error bars $=$ standard error of the mean. (c) Immunoblots show luc-Hep3B tumors with GAPDH shRNA-dependent decrease in GAPDH and a corresponding increase in caspase- 9 and active caspase- 3 levels. Although GAPDH shRNA showed a decrease in phosphorylated Akt, shRNA treatment differed significantly from 3-BrPA treatment by not inducing phosphorylated heat shock protein (HSP) 27. Pin1 = loading control. Numbers in parentheses in the immunoblots (in c) correspond to the respective after treatment (Post- $T x$ ) mouse on bioluminescence images (in a). Numbers at the bottom of blots are densitometry quantifications of respective signals. $p=$ photons, $p / s=$ photons per second, $s r=$ steradian. 


\section{Figure 6}

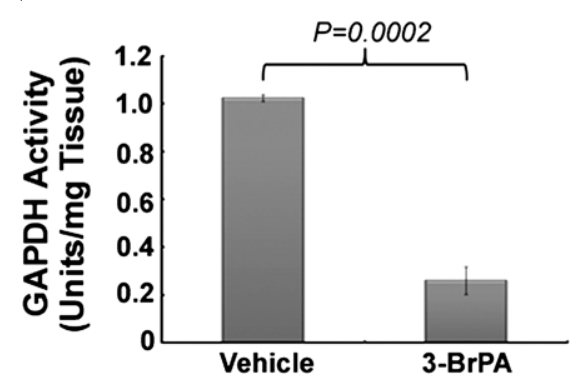

a.

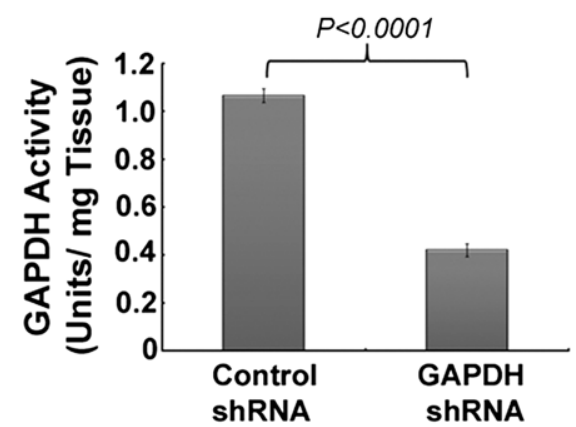

c.

Figure 6: Graphs show that percutaneous injection of 3-BrPA or GAPDH shRNA affects GAPDH activity and expression. (a) 3-BrPA (1.75 mmol/L) treatment significantly reduced mean GAPDH activity $(n=6)$. (b) Mean GAPDH mRNA level showed a marked decrease in 3-BrPA (1.75 mmol/L)-treated tumor $(n=6)$. (c) GAPDH shRNA treatment significantly reduced mean GAPDH activity $(n=6)$. (d) Mean GAPDH mRNA level showed a significant decrease in GAPDH shRNA-treated tumor $(n=6)$. Error bars = standard error of the mean.

the therapeutic target, GAPDH, both showed a decrease in their intensity in the treated (light yellowish-brown with altered tissue architecture) compared with the control (dark brown stain with intact cells) tumors.

\section{GAPDH Is Frequently \\ Upregulated in Human HCC}

Analysis of HCC samples from 34 patients showed elevated levels of GAPDH expression (range, 2.0 to 100 fold) in $68 \%$ (23 of 34 ) of cases compared with the matched nonneoplastic tissues (Fig 8a), and the protooncogene $c$-jun showed upregulation in $59 \%$ (20 of 34 ) of cases. Spearman rank correlation coefficient showed that GAPDH mRNA positively correlated with the expression of $c$-jun mRNA $(r=0.543, P=.003)$, indicating its role in HCC (Fig 8b). Together, our data indicate GAPDH is upregulated in human HCC, and molecular targeting of GAPDH either by using an inhibitor or shRNA results in apoptosis. Figure 8c represents a schematic diagram showing the potential antitumor effects of targeting GAPDH in human HCC.

\section{Discussion}

Our results unequivocally demonstrated that percutaneous injection of GAPDH antagonists such as 3-BrPA or GAPDH shRNA block the progression of luc-Hep3B tumor in a mouse model. Characterization of the therapeutic response by using bioluminescence imaging and biochemical analysis confirmed that the blockade of tumor progression was achieved by targeted inhibition of GAPDH that resulted in the induction of apoptosis. Furthermore, analysis of HCC from patient samples revealed that the incidence of GAPDH upregulation in human HCC strongly correlates with $c$-jun, a proto-oncogene that has already been known to be involved in liver tumorigenesis (22). It remains to be seen if such a correlation exists between GAPDH expression and other oncogenes (eg, KRAS) in HCC progression. Thus, our findings indicated that GAPDH could be a target for therapeutic intervention and the percutaneous or locoregional administration of GAPDH antagonists successfully targets GAPDH, causing tumor cell death.

Our results also demonstrated that the primary effect of $3-\mathrm{BrPA}$ is on the activity of GAPDH (74.5\% inhibition) compared with shRNA-mediated inactivation $(60.6 \%)$, whereas the primary effect of GAPDH shRNA is on the expression of GAPDH mRNA (44.4\% knockdown compared with 3-BrPA-mediated depletion of $34.3 \%$ ). Thus, the primary effect of either GAPDH inactivation or its depletion depends on the therapeutic agent.

Ever since Warburg (23) demonstrated the prevalence of aerobic glycolysis in tumor cells, several glycolytic targets were tested for the disruption of energy metabolism in cancer cells. Enzymes such as lactate dehydrogenase (24) and hexokinase II (25) have been investigated in preclinical models of HCC as potential targets for therapy, without any success in clinical trials. Hence, the need to identify a target that is critical for tumor growth but sensitive to therapy remains. GAPDH, apart from its glycolytic function, is also known to be involved in multiple processes such as chemoresistance $(26,27)$, metastatic potential (28), protection against caspase-independent cell death (29), and cell cycle (15). Inhibition of GAPDH is anticipated to surpass the effect of a single molecule (eg, lactate dehydrogenase, hexokinase II) targeted therapy that exclusively attacks one molecule, hence one pathway or function, where the interrupted physiology could be compensated by collateral or alternative mechanisms. Conceivably, 


\section{Figure 7}
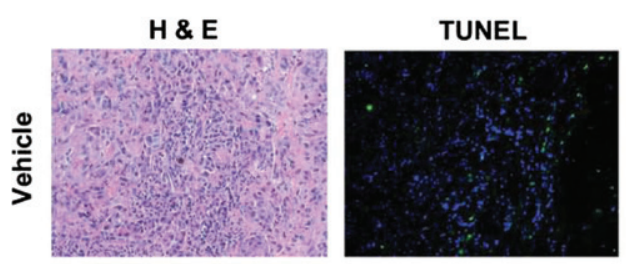

Active caspase-3
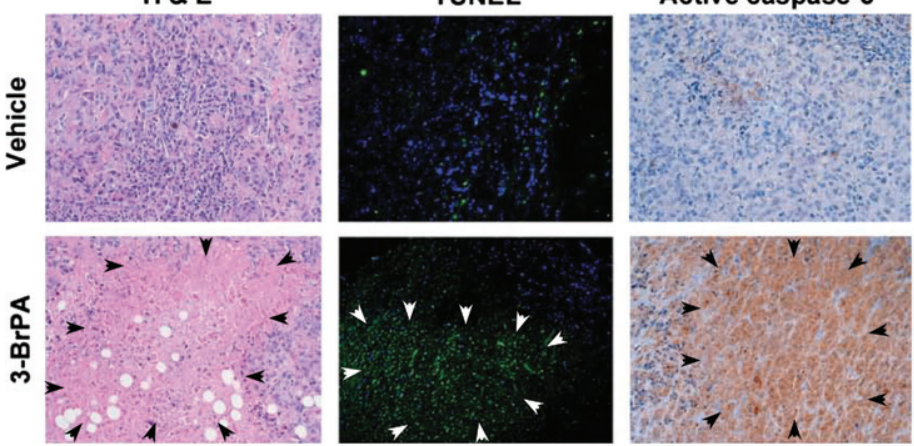

a.
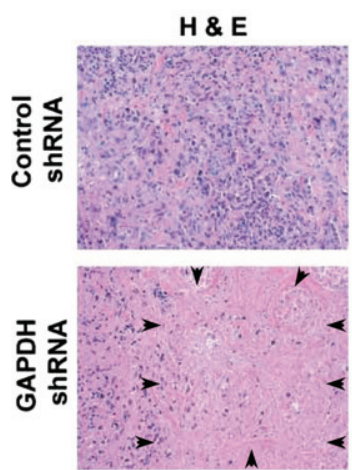

b.

Figure 7: Percutaneous injection of 3-BrPA or GAPDH shRNA affects tumor by inducing apoptosis. Photomicrographs of representative tumor slices from (a) control (vehicle) and 3-BrPA-treated and (b) control shRNA and GAPDH shRNA-treated mice. Hematoxylin-eosin (H \& E) staining shows a dose-dependent alteration in tumor histologic findings, as evident from the differential staining of nucleus (purple) and cytoplasm (pink). TUNEL staining shows positive signal (green fluorescence) with fluorescent microscopy, demonstrating the induction of apoptosis with 3-BrPA and GAPDH shRNA treatments. 4',6-diamidine-2-phenylindole nuclear staining (blue) was used as a counterstain for viable cells. Immunohistochemical staining shows the activation of caspase-3 (dark brown) in treated tumors. Hematoxylin counterstain (blue) is more abundant in control (vehicle or control shRNA) than in treated tumors. Immunohistochemical staining shows a decrease in the staining intensity of $\alpha$-fetoprotein (AFP) (light brown) and GAPDH (light brown) in a treatment-dependent manner, counterstained with hematoxylin (blue). Arrowheads $=$ treatment-dependent histologic alterations in the tumor. (Hematoxylin-eosin stain; original magnification, $\times 100$. Immunohistochemical stain; original magnification, $\times 100$. TUNEL stain; original magnification, $\times 40$.)
GAPDH inhibition could sensitize the cancer cells for chemotherapy, because the protection and resistance offered by GAPDH will be abrogated. Thus, targeted inhibition of GAPDH by using a local or intratumoral approach would enable us to overcome the current challenges in chemotherapy.

Although the therapeutic potential of RNA interference strategy for treating HCC has been increasingly recognized (30), it remains largely unexplored to our knowledge, and our data document the plausibility of shRNAmediated GAPDH knockdown in the management of HCC. However, two major challenges to this approach are the ubiquitous nature of GAPDH, raising the concern for nonspecific toxicity, and its intracellular abundance, which would require higher doses of drug for therapeutic effect. To overcome these challenges, locoregional therapies such as percutaneous and intraarterial deliveries provide a viable alternative to systemic administration $(31,32)$ because of the unique advantage of selectively targeting tumors with image guidance while minimizing systemic toxicity (3). Our results demonstrated that percutaneous injection of GAPDH antagonists block HCC progression. Although our data did not show complete disappearance or death of tumor in all the animals under the current treatment regimen, the present report certainly documented the therapeutic response of the tumor to GAPDH inhibition. Further studies on the optimization of treatment regimen as well as delivery system with GAPDH antagonists will enable us to characterize the means of achieving maximal therapeutic efficacy either alone or in combination with other therapies. Because 


\section{Figure 8}

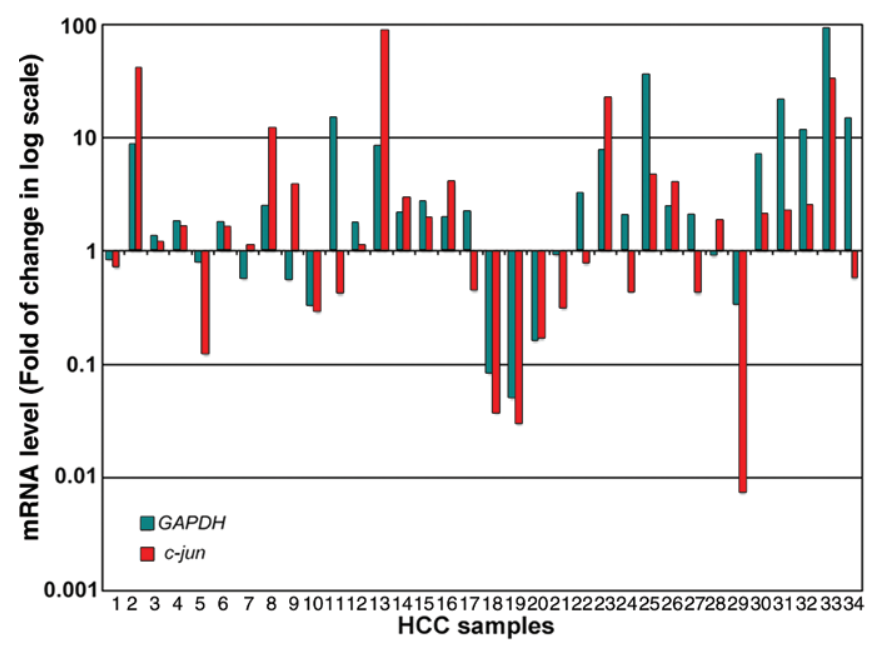

a.

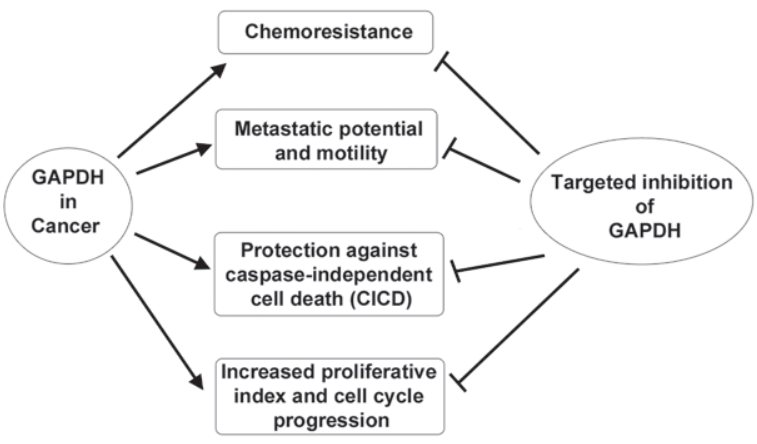

nanoparticle-based slow release of potential therapeutic agents has shown promising results in preclinical models $(33,34)$, integration of such technology and anti-GAPDH approach will allow us to improve the efficacy of therapy with minimal number of percutaneous injections, which is feasible in clinical translation. In summary, molecular targeting of GAPDH through percutaneous injection of either an inhibitor, 3-BrPA, or shRNA blocks tumor progression, which demonstrates the therapeutic potential of targeting GAPDH in HCC.

Practical applications: Molecular targeting of GAPDH has the potential to block tumor progression in human HCC, and our results demonstrated that such a blockade involves induction of apoptotic pathway. We further demonstrated that percutaneous injection of GAPDH antagonists (eg, 3-BrPA or
GAPDH shRNA) could be very effective in achieving tumor kill, and such agents may have the advantage of easy translation to clinics with current locoregional therapies.

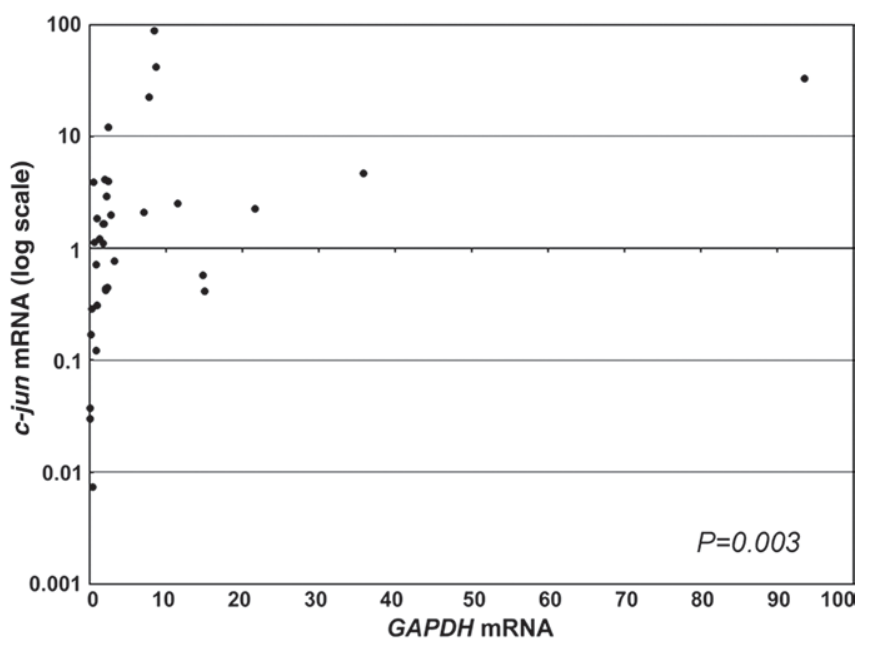

b.

Figure 8: GAPDH is frequently overexpressed in human HCC. (a) Graph shows real-time polymerase chain reaction analysis of HCC samples normalized with matched paraneoplastic tissues showing overexpression of GAPDH in 68\% (23 of 34) of cases and $c$-jun in 59\% of cases. (b) Spearman rank correlation coefficient showed significant positive correlation between GAPDH and c-jun mRNA expression $(P=.003)$. (c) Diagram shows anticancer advantages of targeting GAPDH. Arrows = involvement of GAPDH. Bars = abrogation of the role of GAPDH.

\section{References}

1. Yang JD, Roberts LR. Hepatocellular carcinoma: a global view. Nat Rev Gastroenterol Hepatol 2010;7(8):448-458.

2. Marin JJ, Romero MR, Briz O. Molecular bases of liver cancer refractoriness to pharmacological treatment. Curr Med Chem 2010;17(8):709-740.

3. Lencioni R. Loco-regional treatment of hepatocellular carcinoma. Hepatology 2010;52(2): $762-773$.

Acknowledgments: We thank Zaver Bhujwalla, $\mathrm{PhD}$, Arvind Pathak, PhD, and Scott Kominsky, $\mathrm{PhD}$, for allowing us to use the Victor3 plate reader, fluorescent microscopy, and spectrophotometer, respectively.

Disclosures of Potential Conflicts of Interest: S.G.K. No potential conflicts of interest to disclose. R.K. No potential conflicts of interest to disclose. M.S.T. No potential conflicts of interest to disclose. P.P.R. No potential conflicts of interest to disclose. K.A.C. No potential conflicts of interest to disclose. M.B. No potential conflicts of interest to disclose. M.V. No potential conflicts of interest to disclose. J.F.H.G. Financial activities related to the present article: none to disclose. Financial activities not related to the present article: author is consultant for Biocompatibles, Bayer HealthCare, Guerbet, and Nordion; institution has grants or grants pending from Biocompatibles, Genentech, Bayer HealthCare, Philips Medical, Nordion, Context Vision, CeloNova. Other relationships: none to disclose.
4. Liapi E, Geschwind JF. Transcatheter and ablative therapeutic approaches for solid malignancies. J Clin Oncol 2007;25(8):978-986.

5. Liapi E, Geschwind JF. Intra-arterial therapies for hepatocellular carcinoma: where do we stand? Ann Surg Oncol 2010;17(5): 1234-1246.

6. Ahmed M, Brace CL, Lee FT Jr, Goldberg SN. Principles of and advances in percutaneous ablation. Radiology 2011;258(2):351-369.

7. Naitoh Y, Kawauchi A, Mizutani Y, et al. Significant antitumor effect of intratumoral ethanol injection on renal cell carcinoma. Eur Urol 2003;44(4):452-457.

8. Yoon DJ, Kwan BH, Chao FC, et al. Intratumoral therapy of glioblastoma multiforme using genetically engineered transferrin 
for drug delivery. Cancer Res 2010;70(11): $4520-4527$.

9. Veltri A, Moretto P, Doriguzzi A, Pagano E, Carrara G, Gandini G. Radiofrequency thermal ablation (RFA) after transarterial chemoembolization (TACE) as a combined therapy for unresectable non-early hepatocellular carcinoma (HCC). Eur Radiol 2006; 16(3):661-669.

10. Dang CV, Hamaker M, Sun P, Le A, Gao P. Therapeutic targeting of cancer cell metabolism. J Mol Med (Berl) 2011;89(3): 205-212.

11. Hugo-Wissemann D, Anundi I, Lauchart W, Viebahn R, de Groot H. Differences in glycolytic capacity and hypoxia tolerance between hepatoma cells and hepatocytes. Hepatology 1991;13(2):297-303.

12. Gong Y, Cui L, Minuk GY. Comparison of glyceraldehyde-3-phosphate dehydrogenase and 28s-ribosomal RNA gene expression in human hepatocellular carcinoma. Hepatology 1996;23(4):734-737.

13. Lau WY, Lai PB, Leung MF, et al. Differential gene expression of hepatocellular carcinoma using cDNA microarray analysis. Oncol Res 2000;12(2):59-69.

14. Kim JW, Kim TE, Kim YK, et al. Antisense oligodeoxynucleotide of glyceraldehyde3-phosphate dehydrogenase gene inhibits cell proliferation and induces apoptosis in human cervical carcinoma cell lines. Antisense Nucleic Acid Drug Dev 1999;9(6):507513.

15. Phadke MS, Krynetskaia NF, Mishra AK, Krynetskiy E. Glyceraldehyde 3-phosphate dehydrogenase depletion induces cell cycle arrest and resistance to antimetabolites in human carcinoma cell lines. J Pharmacol Exp Ther 2009;331(1):77-86.

16. Sirover MA. Role of the glycolytic protein, glyceraldehyde-3-phosphate dehydrogenase, in normal cell function and in cell pathology. J Cell Biochem 1997;66(2):133-140.
17. Ganapathy-Kanniappan S, Geschwind JF, Kunjithapatham R, et al. Glyceraldehyde3 -phosphate dehydrogenase (GAPDH) is pyruvylated during 3-bromopyruvate mediated cancer cell death. Anticancer Res 2009;29(12):4909-4918.

18. Ray P, De A, Min JJ, Tsien RY, Gambhir SS. Imaging tri-fusion multimodality reporter gene expression in living subjects. Cancer Res 2004;64(4):1323-1330.

19. Franken NA, Rodermond HM, Stap J, Haveman J, van Bree C. Clonogenic assay of cells in vitro. Nat Protoc 2006;1(5):2315-2319.

20. Bergmeyer HU, Bergmeyer J, Grassl M. Methods of enzymatic analysis. 3rd ed. Weinheim, Germany: Verlag Chemie, 1983.

21. Vali M, Liapi E, Kowalski J, et al. Intraarterial therapy with a new potent inhibitor of tumor metabolism (3-bromopyruvate): identification of therapeutic dose and method of injection in an animal model of liver cancer. J Vasc Interv Radiol 2007;18(1 pt 1):95-101.

22. Eferl R, Ricci R, Kenner L, et al. Liver tumor development: c-Jun antagonizes the proapoptotic activity of p53. Cell 2003;112(2): 181-192.

23. Warburg O. On respiratory impairment in cancer cells. Science 1956;124(3215):269270.

24. Fiume L, Manerba M, Vettraino M, Di Stefano G. Impairment of aerobic glycolysis by inhibitors of lactic dehydrogenase hinders the growth of human hepatocellular carcinoma cell lines. Pharmacology 2010;86(3):157-162.

25. Ahn KJ, Hwang HS, Park JH, et al. Evaluation of the role of hexokinase type II in cellular proliferation and apoptosis using human hepatocellular carcinoma cell lines. J Nucl Med 2009;50(9):1525-1532.

26. Demarse NA, Ponnusamy S, Spicer EK, et al. Direct binding of glyceraldehyde 3-phosphate dehydrogenase to telomeric DNA protects telomeres against chemother- apy-induced rapid degradation. J Mol Biol 2009;394(4):789-803.

27. Hulleman E, Kazemier KM, Holleman A, et al. Inhibition of glycolysis modulates prednisolone resistance in acute lymphoblastic leukemia cells. Blood 2009;113(9):20142021.

28. Epner DE, Partin AW, Schalken JA, Isaacs JT, Coffey DS. Association of glyceraldehyde-3-phosphate dehydrogenase expression with cell motility and metastatic potential of rat prostatic adenocarcinoma. Cancer Res 1993;53(9):1995-1997.

29. Colell A, Ricci JE, Tait S, et al. GAPDH and autophagy preserve survival after apoptotic cytochrome $\mathrm{c}$ release in the absence of caspase activation. Cell 2007;129(5):983-997.

30. Xu C, Lee SA, Chen X. RNA interference as therapeutics for hepatocellular carcinoma. Recent Patents Anticancer Drug Discov 2011;6(1):106-115.

31. Hong K, Geschwind JF. Locoregional intra-arterial therapies for unresectable intrahepatic cholangiocarcinoma. Semin Oncol 2010;37(2):110-117.

32. Liapi E, Geschwind JF. Interventional oncology: new options for interstitial treatments and intravascular approaches-targeting tumor metabolism via a loco-regional approach: a new therapy against liver cancer. $\mathrm{J}$ Hepatobiliary Pancreat Sci 2010;17(4):405406.

33. Xu Y, Chenna V, Hu C, et al. A polymeric nanoparticle encapsulated hedgehog pathway inhibitor HPI-1 (NanoHHI) inhibits systemic metastases in an orthotopic model of human hepatocellular carcinoma. Clin Cancer Res doi: 10.1158/1078-0432. Published online August 25, 2011.

34. Zhou Q, Ching AK, Leung WK, et al. Novel therapeutic potential in targeting microtubules by nanoparticle albumin-bound paclitaxel in hepatocellular carcinoma. Int $\mathrm{J}$ Oncol 2011;38(3):721-731. 